\title{
Leiomiosarcoma primario de páncreas. A propósito de un caso
}

\author{
Guillermo Carnevale, ${ }^{1}$ Adolfo Wulfson, ${ }^{2}$ Claudio Guerrina ${ }^{3}$ \\ ${ }^{1}$ Hospital Italiano Rosario (H.I.R.). Rosario, Argentina. \\ ${ }^{2}$ Docente carrera de Postgrado en Gastroenterología y Endoscopia de la Facultad de Ciencias Médicas, Rosario (U.N.R.). \\ Gastroenterología Rosario (GR). Argentina. \\ ${ }^{3}$ Hospital Dr. Clemente Álvarez (H.E.C.A.), Hospital Provincial Centenario. Rosario, Argentina. \\ Colaboradores: Guillermo D’ottavio, ${ }^{4}$ Nidal Mawuasi, ${ }^{5}$ Miguel Muñoz, ${ }^{6}$ Germán Sánchez Granel ${ }^{7}$ \\ ${ }^{4}$ Gastroenterólogo, (H.I.G.). Rosario, Argentina. \\ ${ }^{5}$ Gastroenterólogo. Haifa, Israel. \\ ${ }^{6}$ Oncología clínica, Centro oncológico de Rosario. Rosario, Argentina. \\ ${ }^{7}$ Quantum, Anatomía patológica. Rosario, Argentina.
}

Acta Gastroenterol Latinoam 2020;50(3):330-334

Recibido: 04/04/2018 / Aceptado: 07/07/2020 / Publicado online: 28/09/2020

\section{Resumen}

El leiomiosarcoma primario de páncreas es un tumor infrecuente. Es probable que su origen sea el músculo liso de los conductos y la pared de los pequeños vasos pancreáticos. Actualmente, son pocos los casos publicados en la literatura mundial. Dada su infrecuencia, existen escasos datos que describan con precisión sus características epidemiológicas, su evolución y las estrategias terapéuticas. Un elemento común lo constituye el mal pronóstico y el comportamiento agresivo con metástasis temprana por la vía hematógena. Reporte de caso. Un varón de 61 años que consulta por dolor abdominal y pérdida de peso. En la tomografía computada abdominal se observa una masa sólida voluminosa que compromete el cuerpo y la cabeza del páncreas y múltiples lesiones hepáti- cas nodulares sólidas compatibles con metástasis. Anatomía patológica. Leiomiosarcoma primario de páncreas, (+) para caldesmón, actina músculo liso y músculo específica. Se inicia quimioterapia y luego de 27 meses el paciente fallece debido a la progresión de su enfermedad. Conclusión. El leiomiosarcoma primario de páncreas, representa una neoplasia poco frecuente, con características clínicas e imagenológicas inespecíficas. El diagnóstico inmunohistoquímico resulta ser esencial, debido a los diversos diagnósticos diferenciales. Es una neoplasia de mal pronóstico debido a su comportamiento agresivo y las metástasis hematógenas tempranas.

Palabras claves. Leiomiosarcoma primario de páncreas, tumores infrecuentes de páncreas, páncreas.

\section{Primary leiomyosarcoma of the pancreas. A case report}

\section{Summary}

Primary Pancreatic Leiomyosarcoma is a rare tumour. It probably originates from the smooth muscle of the pancreatic ducts or the small pancreatic vessels. Given its rarity and the scarce published cases, only few data are available regarding its epidemiological characteristics, evolution and therapeu- 
tic strategies. Common elements of the previously published cases were poor prognosis and aggressive course with early haematogenous metastasis. Case report. A 61-year-old man presented with subacute abdominal pain and weight loss. Abdominal computerized tomography showed a large solid mass that encompasses the body and head of the pancreas in addition to multiple solid nodular lesions in the liver compatible with metastases. Pathology report. Primary pancreatic leiomyosarcoma, immunologically-stained positive for caldesmon, smooth muscle actin. Chemotherapy was given with initial improvement. The patient survived for 27 months. Conclusion. The rarity and the ominous course of primary pancreatic leiomyosarcoma impose great medical challenge. It also lacks specific clinical and imaging characteristics and evidence-based treatment strategy. The immunohistochemically diagnosis proves to be essential, due to the important differential diagnosis. Its ominous prognosis corresponds to its aggressive course and early haematogenous metastases.

Key words. Primary leiomyosarcoma of the pancreas, rare tumors of the pancreas, pancreas.

\author{
Abreviaturas \\ AP: Anatomía patológica. \\ VEDA: Videoendoscopia digestiva alta. \\ IGV 1: Várices gástricas aisladas. \\ HPT: Hipertensión portal. \\ AFP: Alfa feto proteina. \\ CEA: Antígeno carcinoembrionario. \\ FMO: Falla multiorgánica. \\ IHQ: Inmunohistoquimica. \\ AML: Actina de musculo liso. \\ AME: Actina de músculo especifica. \\ EBV: Epstein-Barr virus. \\ TC: Tomografía computada.
}

\section{Introducción}

El leiomiosarcoma primario (LMP) de páncreas es un tumor infrecuente. Es probable que su origen sea el músculo liso de los conductos y la pared de los pequeños vasos pancreáticos. Hasta la actualidad se han publicado entre 52 y 64 casos en la literatura inglesa. ${ }^{1,2}$ Dada su infrecuencia, existen escasos datos que describan con precisión sus características epidemiológicas, su evolución y las estrategias terapéuticas. Un elemento común lo constituye el mal pronóstico y el comportamiento agresivo con metástasis tempranas por la vía hematógena.

El objetivo de esta publicación es presentar un caso de lesión pancreática infrecuente con revisión de la literatura.

\section{Caso clínico}

Paciente varón, de 61 años de edad, consultó en febrero del año 2014 con un cuadro de dolor abdominal de reciente comienzo y pérdida de peso. Ingesta de alcohol de $70 \mathrm{~g} / \mathrm{d}$ por 20 años, con exposición a plaguicidas de forma prolongada. No presentaba antecedentes familiares relevantes. En el examen físico, hepatomegalia a 3 traveses del reborde costal, de consistencia duro elástica y con borde irregular e ictericia cutánea mucosa. El resto del examen es normal. Dado el inicio reciente de los síntomas y el antecedente de etilismo, se realiza una VEDA observándose en fundus gástrico un cordón tortuoso único de coloración azulado compatible con una várice fúndica aislada (IGV 1) de la clasificación de Sarín (Figura 1).

\section{Figura 1. Hallazgo endoscópico de una várice fúndica aislada}

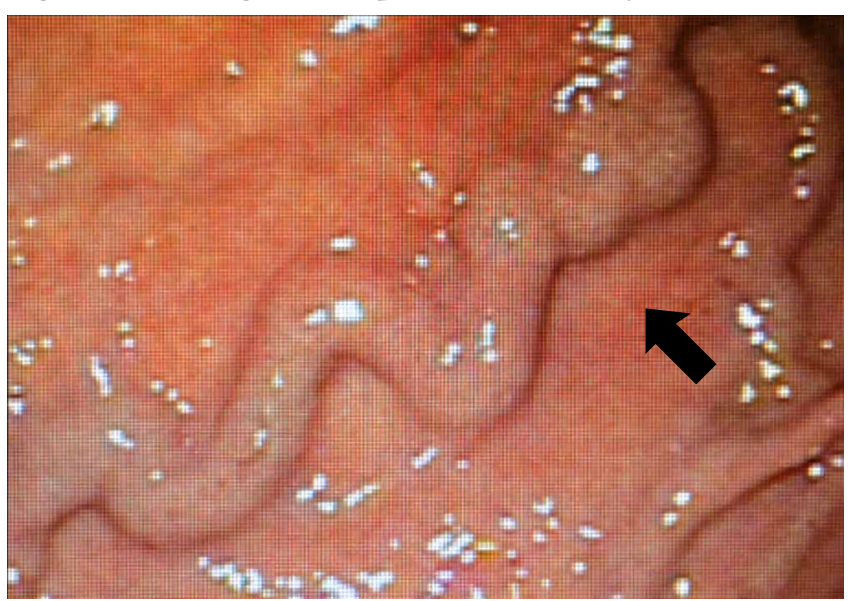

Se solicitan estudios bioquímicos. Los resultados son: Htco 30\%, plaquetas: 156000, GOT $124 \mathrm{UI} / \mathrm{ml}$, GPT $250 \mathrm{UI} / \mathrm{ml}$, FAL: $770 \mathrm{UI} / \mathrm{ml}$, GGT: $400 \mathrm{UI} / \mathrm{ml}$, BT: $6 \mathrm{~g} / \mathrm{dl}, \mathrm{BD}: 3,4 \mathrm{~g} / \mathrm{dl}$, amilasa: $78 \mathrm{UI} / \mathrm{L}$ y albúmina 3,5 g/l. La ecografía abdominal informó hepatomegalia con bordes irregulares y presencia de múltiples nódulos sólidos hipoecoicos. En el páncreas se observó una formación hipoecoica irregular de $5 \times 5 \mathrm{~cm}$ y el bazo normal. Se solicitó un RMI de abdomen donde se informó un voluminoso efecto de masa sólido comprometiendo el cuerpo y la cabeza del páncreas, de $10 \times 8 \mathrm{~cm}$ y que se extendía hasta el tejido graso peripancreático. Además, se observaron múltiples lesiones hepáticas nodulares sólidas con captación periférica del contraste y un lavado rápido en fase venosa tardía. La masa pancreática provocaba la obstrucción del eje esplenoportal, invadiendo la vena esplénica (Figuras 2 y 3 ).

Hasta aquí se interpretó el cuadro clínico como una enfermedad oncológica con fuerte sospecha de ser primaria del páncreas, con compromiso hepático y del eje esplenoportal generando HPT regional. Los marcadores 
Figura 2 2y 3. RMI: A) Se observa un compromiso de la porción cefálica pancreática y múltiples lesiones hepáticas. B) Se puede ver una trombosis del eje esplenoportal

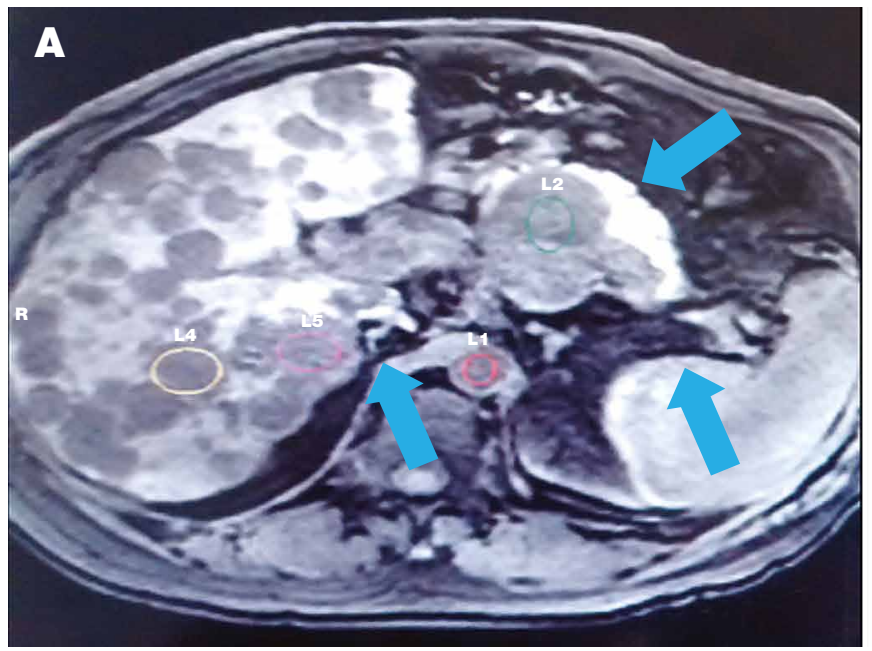

tumorales CA 19-9, AFP, CEA y también el ácido 5-hidroxiindolacético fueron normales. Se realizó una biopsia pancreática guiada por TC. AP: leiomiosarcoma primario de páncreas con caldesmón y desmina $(+)$.

Inició tratamiento con doxorrubicina y dacarbazina (15 ciclos) con buen estado general, asintomático, anictérico y ganancia ponderal. A los 15 meses del diagnóstico se realizó una TC de control donde se observó una leve mejoría en relación a las lesiones hepáticas previas como también del compromiso pancreático (Figuras 4 y 5 ). Además, se realizó punción hepática con el objetivo de reevaluar histológicamente las mismas (Figura 6).

Luego de 27 meses del diagnóstico presenta una ascitis hemorrágica falleciendo con un cuadro de sepsis y FMO.

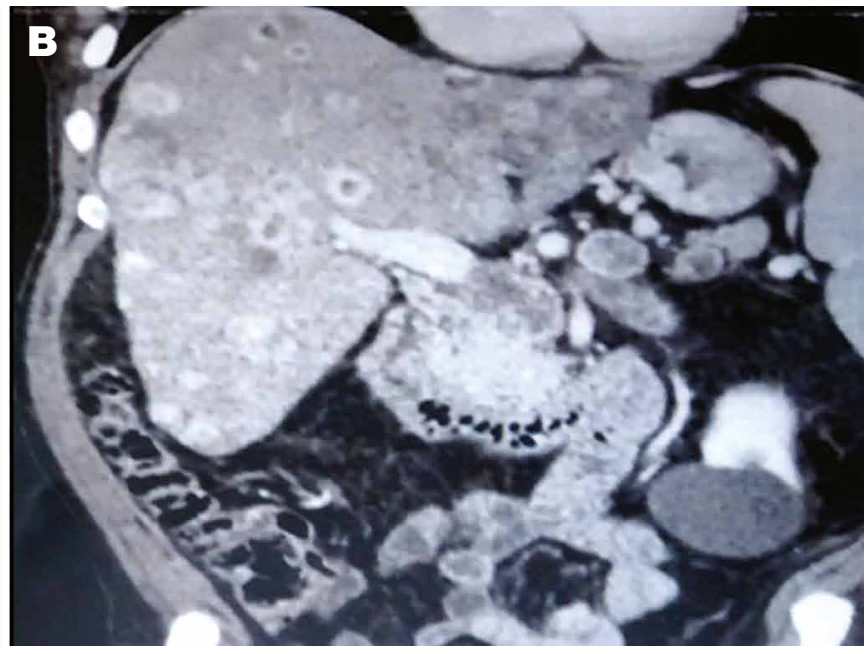

\section{Discusión}

El LMS pancreático es un tumor mesenquimal infrecuente que se origina en el tejido muscular liso presente en las estructuras del páncreas (ductos y paredes vasculares). ${ }^{3}$ Baylor $e t$ al. demostraron la presencia de solo cinco leiomiosarcomas entre 5057 tumores malignos de páncreas examinados. ${ }^{4}$ A pesar de su infrecuencia, constituye la forma más frecuente de sarcomas primarios del páncreas. ${ }^{5}$

La edad promedio de presentación es 53 años, con igual distribución entre los hombres y las mujeres. ${ }^{6}$ Clínicamente, se manifiesta con síntomas inespecíficos como dolor abdominal y pérdida de peso. Constituyen masas voluminosas con degeneración quística, necrosis y hemorragia, afectando a toda la glándula por igual. ${ }^{7}$ Con res-

Figura 4 y 5. Disminución del tamaño tanto de la lesión pancreática como de las metástasis hepáticas

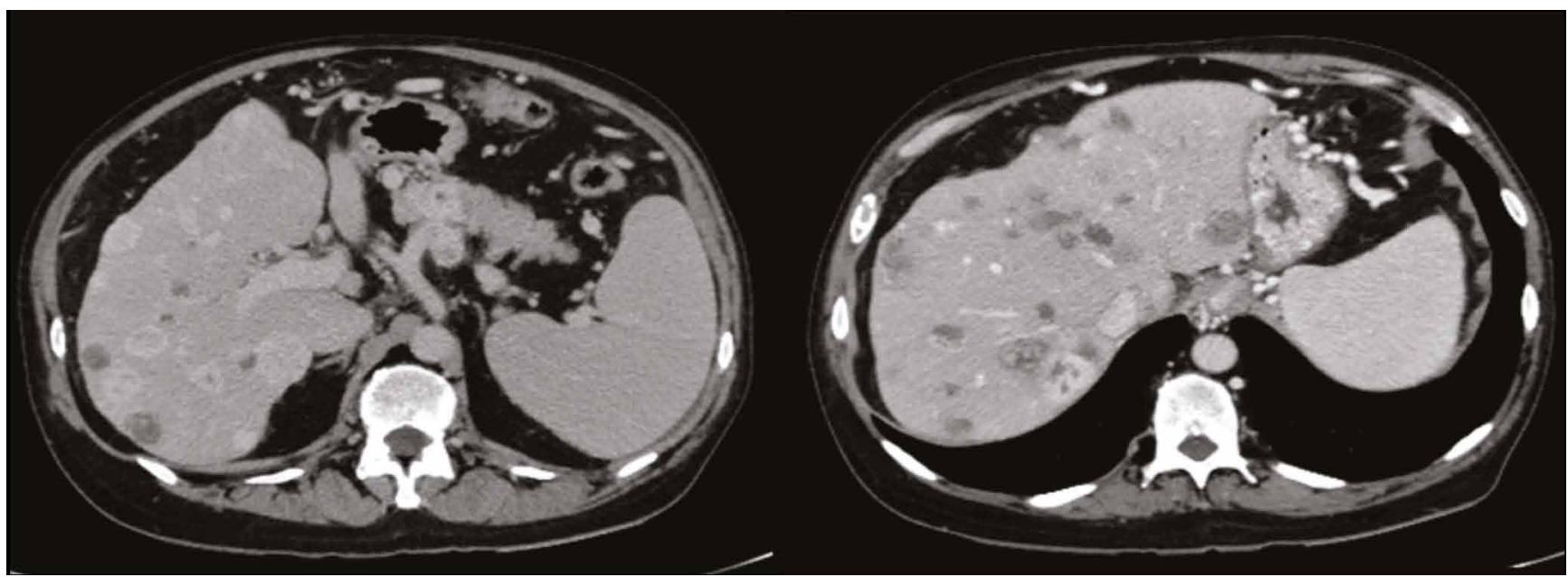


Figura 6. Anatomía patológica e IHQ de las metástasis hepáticas. A) H\&E 100x: se observa un patrón fascicular de distribución de las células fusadas tumorales con pequeñas hendiduras correspondientes a su red vascular. B) H\&E 400x: se pueden ver células tumorales fusocelulares, algunas pleomórficas, con núcleos en forma de "cigarro", hipercromáticos, con variable atipia, citoplasma granular y distribución fascicular. $\boldsymbol{C} \boldsymbol{y}$ D) Marcación actina. Se observa tinción de membrana y citoplasmática en las células tumorales
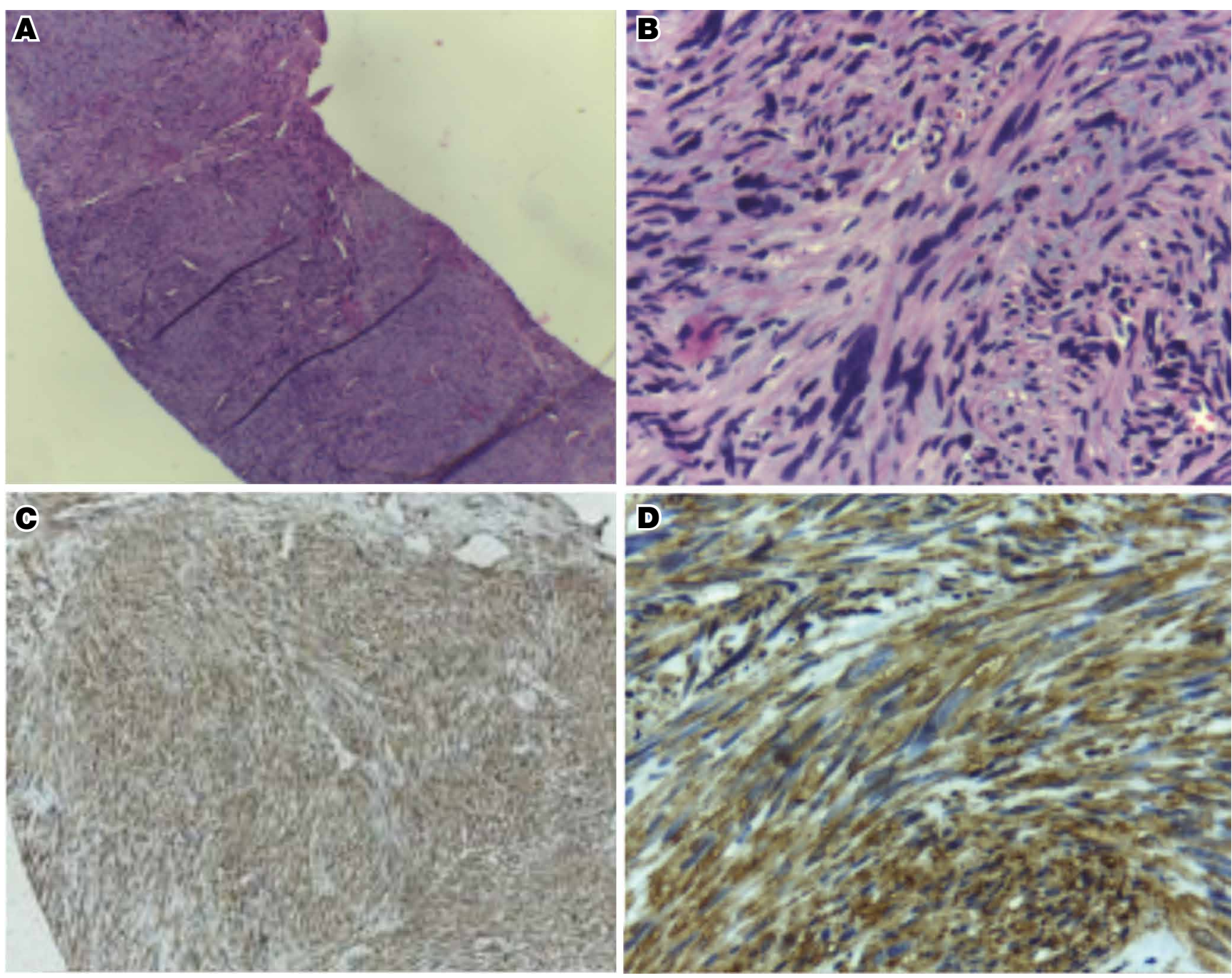

pecto al diagnóstico por imágenes, no presenta características morfológicas específicas, pudiendo generarse dificultades para diferenciarlo, incluso de pseudoquistes pancreáticos. ${ }^{8}$ Son tumores de comportamiento agresivo. Una revisión mostró la existencia de metástasis a distancia hasta en el 25\% de los pacientes al momento del diagnóstico y hasta en un $19 \%$ con compromiso de órganos vecinos. ${ }^{9}$ Se diseminan principalmente por vía hematógena, siendo el compromiso linfático infrecuente. ${ }^{10}$ Es importante destacar la capacidad para simular otras neoplasias mesenquimales y no mesenquimales. Este hecho obliga a realizar un exhaustivo estudio IHQ e incluso molecular. ${ }^{10}$ En tal aspecto, se caracterizan por expresar al menos 2 marcadores de músculo liso (AML y AME, caldesmón, calponina y miosina del músculo liso). ${ }^{11}$ En relación a su evolución, presenta una sobrevida promedio de 48 meses, siendo la imposibilidad de la resección quirúrgica radical el principal factor del mal pronóstico. ${ }^{12}$ El diagnóstico diferencial se debe realizar con los carcinomas sarcomatoides de células fusiformes, tumores del músculo liso asociados a infección por EBV y otros sarcomas fusocelulares o epiteliales incluyendo al GIST (CD $117+) .{ }^{12}$

\section{Conclusión}

El LMS primario de páncreas representa una neoplasia poco frecuente, con características clínicas e imagenológicas inespecíficas. El diagnóstico inmunohistoquímico resulta ser esencial debido a los importantes diagnósticos diferenciales. Es una neoplasia de mal pronóstico debido a su comportamiento agresivo con metástasis hematógenas tempranas. 
Finalmente, resulta importante destacar el diagnóstico endoscópico de las várices fúndicas aisladas. Como sabemos, la presencia de las mismas en pacientes no cirróticos sugiere la posible existencia de HPT regional. En tal sentido, su hallazgo debe motivar el estudio del eje esplenoportal en búsqueda de trombosis venosa, principalmente. En nuestro paciente, dicho hallazgo resultó ser el evento inicial para el diagnóstico final del leiomiosarcoma primario de páncreas.

\section{Sostén Financiero. Ninguno.}

\section{Conflicto de interés. Ninguno.}

\section{Referencias}

1. Søreide JA, Undersrud ES, Al-Saiddi MSS, Tholfsen T, Søreide K. Primary Leiomyosarcoma of the Pancreas - a Case Report and a Comprehensive Review, J Gastrointest Cancer 2016; 47 (4): 358-365.

2. Du X, Cheng Z, Zhou ZG, Zhang MM, Chen ZX. Primary pancreatic leiomyosarcoma: a retrospective analysis of clinical characteristics and prognosis of this rare disease. Hepatogastroenterology 2012; 59 (120): 2644-2649.

3. Nesi G, Pantalone D, Ragionieri I, Amorosi A. Primary leiomyosarcoma of the pancreas: a case report and review of literature. Arch Pathol Lab Med 2001; 125: 152-155.
4. Baylor SM, Berg JW. Cross-classification and survival characteristics of 5,000 cases of cancer of the pancreas. J Surg Oncol 1973; 5 (4): 335-358.

5. Zalatnai A, Kovács M, Flautner L, Sipos B, Sarkady E, Bocsi J. Pancreatic leiomyosarcoma. Case report with immunohistochemical and flow cytometric studies. Virchows Arch 1998; 432 (5): 469-472.

6. Barros A, Linhares E, Valadão M, Gonçalves R, Vilhena B, Gil C, Ramos C. Extragastrointestinal stromal tumors (EGIST): a series of case reports. Hepatogastroenterology 2011; 58 (107-108): 865-888.

7. Izumi $H$, Okada $K$, Imaizumi $T$, Hirabayashi $K$, Matsuyama M, Dowaki S, Tobita K, Makuuchi H. Leiomyosarcoma of the pancreas: report of a case. Surg Today 2011; 41: 1556-1561.

8. Aihara H, Kawamura YJ, Toyama N, Mori Y, Konishi F, Yamada S. A small leiomyosarcoma of the pancreas treated by local excision. HPB (Oxford) 2002; 4 (3): 145-148.

9. Hur YH, Kim HH, Park EK, Seoung JS, Kim JW, Jeong YY, Lee JH, Koh YS, Kim JC, Kim HJ, Cho CK. Primary leiomyosarcoma of the pancreas. J Korean Surg Soc 2011; 81 (Suppl 1): S69-S73.

10. Xu J, Zhang T, Wang T, You L, Zhao Y. Clinical characteristics and prognosis of primary leiomyosarcoma of the pancreas: a systematic review. World Journal of Surgical Oncology 2013, 11: 290.

11. Zhang H, Jensen MH, Farnell MB, Smyrk TC, Zhang L. Primary leiomyosarcoma of the pancreas: study of 9 cases and review of literature. Am J Surg Pathol 2010; 34 (12): 1849-1856.

12. Barros A, Linhares E, Valadão $M$, Gonçalves R, Vilhena B, Gil C, Ramos C. Extragastrointestinal stromal tumors (EGIST): a series of case reports. Hepatogastroenterology 2011; 58 (107-108): 865-868. 